\title{
Solving Highly-dimensional Fractional Oscillators via an Efficient Scheme Based on Adams and Newmark- $\beta$ Algorithms
}

\author{
Yuxiang Chen, Yanmao Chen and Qixian Liu* \\ Department of Mechanics, Sun Yat-sen University, No.135Xingang Road West, Guangzhou 510275, China \\ ${ }^{*}$ Corresponding author
}

\begin{abstract}
In this paper, we employed an efficient numerical scheme by combining Adams and Newmark- $\beta$ algorithms to solve highly-dimensional nonlinear oscillators containing fractional derivatives (FDs). The method solves the original equations directly by incorporating the Adams and Newmark- $\beta$ algorithms to handle FDs and integer derivatives, respectively. It also provides a strategy to avoid nonlinear algebraic equations arising in the Newmark- $\beta$ algorithm. Both analytical estimations and numerical results show that the presented method is second-order accurate. The applicability and efficiency of the presented method are demonstrated by a simple and feasible extension to solve time fractional nonlinear diffusion-wave equations.
\end{abstract}

Keywords-fractional derivative; nonlinear oscillator; volterra integral equation; newmark- $\beta$ method; adams scheme

\section{INTRODUCTION}

Fractional derivatives (FDs) have shown promising potential in wide applications during the recent decades, due to its simplicity and accuracy in modeling many problems arising in viscoelastic materials [1-2], soft matter [3-4], anomalous diffusion [5-6], control theory [7-8], signal and image processing [9-10], to mention a few. Specifically, constitutive modelling of complex viscoelastic materials is one of the most extensive applications of FDs. As viscoelastic damping materials are utilized more and more widely in the vibration suppression design of structures, the oscillation especially nonlinear oscillation systems containing FDs have stimulated the curiosities and interests for many researchers [11-12].

Well-known, it is different to obtain exact analytical solutions for oscillation systems containing FDs, which are usually described by fractional differential equations (FDEs). Numerical solution techniques have attracted most of the interests of researchers. A large group of numerical algorithms take advantage of the fact that, in certain occasions FDEs can be reduced to Volterra integral equations. For this reason, these methods can be generally called as integral equation methods. For instance, Diethelm et al. [13-14] extended the AdamsBashforth-Moulton type predictor-corrector scheme to FDEs. Kumar and Agrawal [15] construct a linear multi-step method to solve the initial value problem of fractional differential equations. Huang [16] analytically estimated that the convergence order of this scheme is at least three. Cao et al. [17] modified the computational efficiency of this multistep method, and proved the stability and convergence of this method. By using the piecewise quadratic interpolation and Simpson method Li et al. [18] design a four order algorithm for fractional differential equations. Yaghoobi [19] proposed a high order precise scheme for variable-order fractional order integrals based on a cubic spline interpolation technique.

In this study, we consider a general nonlinear oscillator with fractional dampings $[1-2,20-23]$

$$
m \ddot{x}+\mu D^{\alpha} x+k x+f(x, \dot{x})=q(t)
$$

here $x$ denotes the displacement of dimension $n, \mathrm{q}(\mathrm{t})$ denotes the external force, $f(x, x)$ is the nonlinear term, and the FD is in the sense of Caputo-type defined as $D^{\alpha} x=\frac{1}{\Gamma(1-\alpha)} \int_{0}^{t} \frac{\dot{x}(\tau)}{(t-\tau)^{\alpha}} d \tau$ where $0<\alpha<1$. When the above-mentioned integral equation methods [13-19] are implemented, Eq. (1) should be firstly converted into another set of equivalent FDEs given as [14]

$$
D^{\lambda} y=G(t, y)
$$

here $y=\left[y_{1}, y_{2}, \Lambda, y_{m}\right]^{T}$ with $y_{1}=x, y_{i+1}=D^{\lambda} y_{i}$, and $\lambda$ is the great common divisor (gcd) of $\alpha$ and 1 , e.g. $\lambda=\operatorname{gcd}(1, \alpha)$. The induced vector variable, $\mathrm{y}$, will be of dimension $m=2 n / \lambda$. The inhomogeneous vector of the same dimension can be given as $G(t, y)=\left[x_{2}, x_{3}, \Lambda, q(t)-x_{1+\alpha / \lambda}\right.$

$$
\begin{aligned}
& =\left[x_{2}, x_{3}, \Lambda, q(t)-x_{1+\alpha / \lambda}\right. \\
& \left.-k x_{1}-f\left(x_{1}, x_{1+1 / \lambda}\right), x_{n+1}, \Lambda\right]^{T} . \text { It should be noted that, }
\end{aligned}
$$

the dimension of Eq. (2) will become quite high as the gcd, i.e., $\lambda$, is relatively small. It may require a huge of computational efforts to calculate the weights of fractional derivatives. Even worse, it will cease to be valid to derive Eq. (2) from (1) because one cannot determine $\lambda$ for the case when $\alpha$ takes an irrational value. To avoid these drawbacks, Edwards [20] is to use a hybrid system where the equations contain the fractional and integer order. Based on the hybrid system, Liu et al [21] suggested an explicit hybrid algorithm by employing the Adams and RungeKutta schemes. 
It may be an alternative choice to implement numerical schemes to avoid the system dimension increasing, especially when it is incapable to convert Eq. (1)to an equivalent one described as (2). This kind of approaches take advantage of the fact that, the fractional and integer time derivatives are usually directly discretized, respectively, for instance the finite element method [23], finite difference method [24], and spectral method [25], to mention a few.

Well-known, Newmark-type methods can be directly applied to solve vibration equations of second order without transforming them into first-order ones. This provides us with a possible way to solve Eq. (1) without converting it to (2). Zhang and Shimizu proposed a numerical scheme for linear fractional oscillator, in which the integer-order time derivative term is approximated by a Newmark type formulations and the fractional derivative is treated by the product trapezoidal method [26]. Galucio et al [27] combined the Gear-scheme and Newmark- $\beta$ method to solve a fractional damped oscillator. Bucher and Pirrotta [28] utilized the constant average acceleration method in conjunction a discrete version of the Grünwald-Letnikov representation of Caputo fractional derivative, to analyze the earthquake responses of a multi-storey building. Deü and Matignon [29] combined the Newmark timeintegration method and a memory-free transformation principle to solve second-order mechanical systems with fractional derivatives.

As we know, it requires solving nonlinear algebraic equations, if the above-mentioned Newmark- $\beta$ type schemes are directly applied to nonlinear fractional oscillators. Generally speaking, it is time-consuming, even scarcely possible to do so. In order to avoid this disadvantage, Spanos and Evangelatos [30] derived an increment equilibrium equation for a fractional nonlinear oscillator system. However, it would double the computational efforts of the fractional weights when deducing the increment of a fractional derivative.

To avoid solving nonlinear algebraic equations without increasing in the computation costs in calculating fractional weights, recently we proposed an efficient numerical scheme by combining the Adams and Newmark-type algorithms [31]. Here in this paper we employed this efficient hybrid scheme for solving both highly-dimensional fractional oscillators. Furthermore, the proposed can be straightforwardly extended to time fractional nonlinear diffusion-wave equations by incorporating a centre finite difference scheme.

\section{SOLUTION PROCEDURES}

When the above method is directly applied to a fractional nonlinear oscillator, it needs solve nonlinear algebraic equations at each of iteration. This section will introduce a strategy to avoid solving nonlinear algebraic equations without loss of computation precision [31].

Our algorithm is based on the analytical property that the initial problem (1) is equivalent in to the Volterra integral equation

$$
x=x\left(t_{0}\right)+\frac{1}{\mu \Gamma(\alpha)} \int_{0}^{t} \frac{1}{(t-\tau)^{1-\alpha}} G(\tau, x, \dot{x}, \ddot{x}) d \tau
$$

where $G(t, x, \dot{x}, \ddot{x}) d \tau=q(t)-m \ddot{x}-k x-f(x, \dot{x})$. It can be separated as

$$
\begin{aligned}
x_{n}= & x_{o}+\frac{1}{\mu \Gamma(\alpha)} \int_{0}^{t_{n-1}} \frac{1}{\left(t_{n}-\tau\right)^{1-\alpha}} G(t, x, \dot{x}, \ddot{x}) d \tau \\
& +\frac{1}{\mu \Gamma(\alpha)} \int_{t_{n-1}}^{t_{n}} \frac{1}{\left(t_{n}-\tau\right)^{1-\alpha}}(q(\tau)-m \ddot{x}-k x) d \tau \\
& -\frac{1}{\mu \Gamma(\alpha)} \int_{t_{n-1}}^{t_{n}} \frac{1}{\left(t_{n}-\tau\right)^{1-\alpha}} f(x, \dot{x}) d \tau \\
& =x_{o}+\frac{1}{\mu \Gamma(\alpha)}\left(I_{n-1}+I_{n}^{1}-I_{n}^{2}\right)
\end{aligned}
$$

One term in Eq. (4) is calculated by the piecewise linear interpolation such as

$$
\begin{aligned}
I_{n}^{1} & =\int_{t_{n-1}}^{t_{n}} \frac{1}{\left(t_{n}-\tau\right)^{1-\alpha}}(q(\tau)-m \ddot{x}-k x) d \tau \\
& =\frac{\Delta t^{\alpha}}{\alpha(\alpha+1)}\left(q\left(t_{n-1}\right)-m \ddot{x}_{n-1}-k x_{n-1}+q\left(t_{n}\right)-m \ddot{x}_{n}-k x_{n}\right)
\end{aligned}
$$

The Newmark-type formula is applied to determine the acceleration in (5)

$$
\ddot{x}_{n}=\frac{1}{\beta \Delta t^{2}}\left(x_{n}-x_{n-1}\right)-\frac{1}{\beta \Delta t} \dot{x}_{n-1}-\left(\frac{1}{2 \beta}-1\right) \ddot{x}_{n-1}
$$

By integrating $I_{n}^{2}$ in Eq. (5) by parts and expanding it as the first-order Taylor series, we obtain

$$
\begin{aligned}
I_{n}^{2} & =\int_{t_{n-1}}^{t_{n}} \frac{1}{\left(t_{n}-\tau\right)^{1-\alpha}} f(x, \dot{x}) d \tau \\
& =\frac{\Delta t^{\alpha}}{\alpha} f\left(x_{n-1}, \dot{x}_{n-1}\right)+\frac{\Delta t^{\alpha}}{\alpha} \int_{t_{n-1}}^{t_{n}}\left(t_{n}-\tau\right)^{\alpha} \dot{f}(x, \dot{x}) d \tau \\
& \approx \frac{\Delta t^{\alpha}}{\alpha} f\left(x_{n-1}, \dot{x}_{n-1}\right)+\frac{\Delta t^{1+\alpha}}{\alpha(\alpha+1)} f\left(x_{n-1}, \dot{x}_{n-1}\right)
\end{aligned}
$$

Note that, there exists no terms associated with $t_{n}$ in the right hand of Eq. (7). That means, it succeeds in avoiding the nonlinear algebraic equation at each of iteration process. Finally, the last term in Eq. (5) $I_{n-1}$ can be calculated as 


$$
\begin{aligned}
I_{n-1} & =\int_{0}^{t_{n}-1} \frac{1}{\left(t_{n}-\tau\right)^{1-\alpha}} G(\tau, x, \dot{x}, \ddot{x}) d \tau \\
& =\frac{\Delta t^{\alpha}}{\alpha(\alpha+1)} \sum_{i=1}^{n-1} \tilde{c}_{i, n} G\left(t_{i}, x_{i}, \dot{x}_{i}, \dot{x}_{i}\right)
\end{aligned}
$$

where

$$
\tilde{c}_{i, n+1}=\left(\begin{array}{lc}
n^{\alpha+1}-(n-\alpha)(n+1)^{\alpha}, & i=0 \\
(n-i+2)^{\alpha+1}+(n-i)^{\alpha+1}-2(n-i+1)^{\alpha+1}, & 1 \leq i \leq n-1 \\
2^{\alpha+1}-\alpha-2, & i=n
\end{array}\right.
$$

Substituting Eqs. (5-8) into (1), we can deduce the following iteration formula

$$
x_{n}=\{\mathrm{B}+\mathrm{C}-\mathrm{D}\} / \mathrm{A}
$$

with $\mathrm{A}=1+\frac{\Delta t^{\alpha}}{\mu \Gamma(2+\alpha)}\left(\frac{m}{\beta h^{2}}+k\right)$

$$
\begin{gathered}
B=x_{0}+\frac{\Delta t^{\alpha}}{\Gamma(2+\alpha)} \sum_{i=1}^{n-1} \tilde{c}_{n, i} G\left(t_{i}, x_{i}, \dot{x}_{i}, \ddot{x}_{i}\right), \\
C=\frac{\Delta t^{\alpha}}{\mu \Gamma(2+\alpha)}\left[\alpha q\left(t_{n-1}\right)+q\left(t_{n}\right)+\left(\frac{m}{\beta \Delta t^{2}}-k\right) x_{n-1}\right. \\
\left.+\frac{m}{\beta \Delta t} \dot{x}_{n-1}+m\left(\frac{1}{2 \beta}-2\right) \ddot{x}_{n-1}\right] \\
D=\frac{\Delta t^{\alpha}}{\mu \Gamma(1+\alpha)}\left[f\left(x_{n-1}, \dot{x}_{n-1}\right)+\frac{\Delta t}{(1+\alpha)} \dot{f}\left(x_{n-1}, \dot{x}_{n-1}\right)\right]
\end{gathered}
$$

After obtaining the displacement by Eq. (9), we can determine the acceleration $\left(\ddot{x}_{n}\right)$ by carrying out iteration formula (6) and the velocity $\left(x_{n}\right)$ by the following formula

$$
\dot{x}_{n}=\frac{\gamma}{\beta \Delta t}\left(x_{n}-x_{n-1}\right)+\left(1-\frac{\gamma}{\beta}\right) \dot{x}_{n-1}+\left(1-\frac{\gamma}{2 \beta}\right) \Delta t \ddot{x}_{n-1}
$$

So far, we have completed the description of our numerical algorithm for fractional nonlinear vibrations.

\section{NUMERICAL EXAMPLES}

In this section, we will present several numerical illustrations to test the accuracy and efficiency of the proposed method. Concretely, the main effort is to verify the convergence order of the numerical solution with respected to the step size $\Delta \mathrm{t}$ and fractional order $\alpha$.

Example I. Consider the free oscillator of linear fractional oscillator. When the initial conditions and system parameters are given as $x\left(t_{0}\right)=0, x\left(t_{0}\right)=x_{0}, m=\mu=k=1$ and $\alpha=0.5$, respectively, the exact solution is [16]

$$
x(t)=\dot{x}_{0} \sum_{i=1}^{4} \frac{\lambda_{i}}{L\left(\lambda_{i}\right)} e^{\lambda_{i}^{2} t}\left[1+\operatorname{erf}\left(\lambda_{i} \sqrt{t}\right)\right]
$$

where $\lambda_{i}(i=1,2,3,4)$ are the root of the characteristic equation of linear fractional oscillator, and $\operatorname{erf}(z)=\frac{2}{\sqrt{\pi}} \int_{0}^{z} e^{-\sigma^{2}} d \sigma$ is the error function and $\dot{L}\left(\lambda_{i}\right)=\Pi_{j \neq i}\left(\lambda_{i}-\lambda_{j}\right)(i, j=1,2,3,4)$.

Figure I reveals the absolution errors induced by the proposed method with time step $\Delta \mathrm{t}=0.01(10-2)$ and different values $\beta$. It is easy to find that the order of absolution errors are about 2 , e.g. $\mathrm{O}(10-4) \approx \Delta t 2$. And the precision slightly increases with the $\beta$ increasing from $1 / 6$ to about 1 .

The comparison of absolution errors caused by the presented method and Zhang-Shimizu algorithm [16], with step size $\Delta \mathrm{t}=0.3$, as shown in Figure II. It can be seen that the presented scheme is more accurate, seen the black quadrates. Notice that, in this case, the presented algorithm is also of second-order accurate.

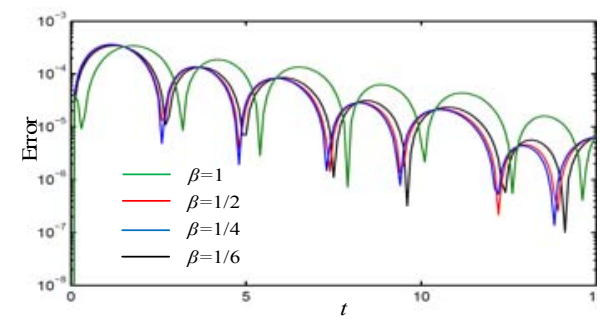

FIGURE I. THE ABSOLUTION ERROR OBTAINED BY PRESENTED METHOD WITH STEP SIZE H=0.01 AND B=1/12 (RED DOTTED LINE), $\mathrm{B}=1$ (BLUE CROSS LINE), $\mathrm{B}=2$ (GREEN TRIANGLE LINE) AND B $=2.5$ (BLACK DASHED LINE) 


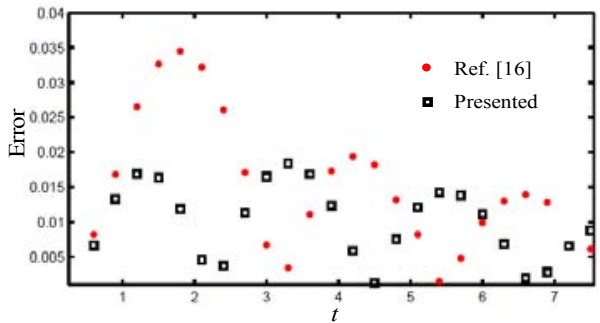

FIGURE II. THE COMPARISON OF THE ABSOLUTION ERROR CAUSED BY PRESENTED METHOD (BLACK SQUARE) AND ZHANG-SHIMIZU METHOD [16] (RED DOTTED) WITH STEP SIZE $\Delta \mathrm{T}=0.3$

Example II. Consider a chain-like n-degree of freedom, which $\mathrm{n}$ different mass $\mathrm{mi}(\mathrm{i}=1,2, \ldots, \mathrm{n})$ connected with each other by fractional Kelvin-Voigt elements. Equations of motion of such a system have the form [32]

$$
\ddot{Z}+U D^{\alpha} z+K z=Q(t)
$$

here, $z=\left[x_{1}, x_{2}, \Lambda, x_{n}\right]^{\mathrm{T}}$ and $Q(t)=\left[q_{1}(t), q_{2}(t), \Lambda, q_{n}(t)\right]^{\mathrm{T}}$ are the $\mathrm{n}$-dimensional vectors. The $n \times n$ matrix $\mu$ and $\mathrm{K}$ have the form

$$
U=\left[\begin{array}{ccccc}
\mu_{1}+\mu_{2} & -\mu_{2} & 0 & 0 & 0 \\
-\mu_{2} & \mu_{2}+\mu_{3} & -\mu_{3} & 0 & 0 \\
0 & \mathrm{O} & \mathrm{O} & \mathrm{O} & 0 \\
0 & 0 & -\mu_{n-1} & \mu_{n-1}+\mu_{n} & -\mu_{n} \\
0 & 0 & 0 & -\mu_{n} & \mu_{n}+\mu_{n+1}
\end{array}\right]
$$

and

$$
K=\left[\begin{array}{ccccc}
k_{1}+k_{2} & -k_{2} & 0 & 0 & 0 \\
-k_{2} & k_{2}+k_{3} & -k_{3} & 0 & 0 \\
0 & \mathrm{O} & \mathrm{O} & \mathrm{O} & 0 \\
0 & 0 & -k_{n-1} & k_{n-1}+k_{n} & -k_{n} \\
0 & 0 & 0 & -k_{n} & k_{n}+k_{n+1}
\end{array}\right]
$$

This example illustrates the accuracy and efficiency of the presented scheme for high-dimensions linear fractional oscillators. Choose the following parameters as $\alpha=0.2, n=10$ and $k_{i}=\mu_{i}=1,(i=1, \Lambda, n)$. Generally, it is very hard to get a closed-form solution for system (21), when each of oscillators is subjected to cosine external forces as $Q(t)=[\cos (t), \cos (t), \Lambda, \cos (t)]^{\mathrm{T}}$.

For the sake of comparison, we display our results and those obtained by the famous predictor-corrector (PC) algorithm [14], as shown in Fig. 3. It is observed that as the time step decrease, the PC approximations gradually tend to the Newmark- $\beta$ solution. Even, the PC algorithm with $\Delta \mathrm{t}=1 / 800$ cannot reach the accuracy of proposed method with $\Delta \mathrm{t}=1 / 100$. This means that the proposed method is much more accurate than the PC algorithm for high-dimensions linear fractional oscillators.

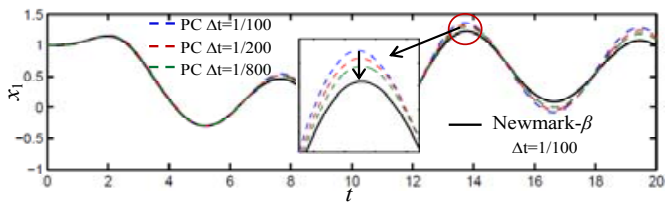

FIGURE III. COMPARISON OF THE TIME HISTORY RESPONSES BETWEEN THE PC AND PROPOSED METHODS FOR APPROXIMATING SYSTEM (11)

In this case, if the PC algorithm is implemented, system (11) should be converted to a set of one hundred dimensions equations because the $\operatorname{gcd}(0.2,1)$ is 0.2 , which is timeconsuming due to computing the abundant additional fractional calculus. Figure IV compares the running time of the proposed method and PC algorithm as a function of the number of grid nodes. It is observed that the running time of $\mathrm{PC}$ algorithm is growing a lot faster than proposed scheme, about ten times.

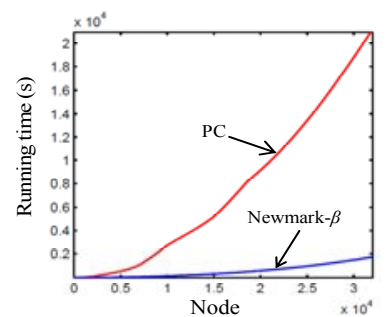

FIGURE IV. COMPARISON OF THE RUNNING TIME BETWEEN THE PC AND PROPOSED METHODS FOR SYSTEM (11)

\section{CONCLUSIONS}

Based on an efficient and accurate scheme that combines the Newmark- $\beta$ method and a modified explicit Adams algorithm, we solved the solution of highly-dimensional linear as well as nonlinear fractional oscillators. The presented method avoids the construction of excessively large systems because the fractional and integer time derivatives are directly discretized. Thus, it can handle with the nonlinear fractional oscillators with arbitrary fractional order, even irrational order. In addition, the proposed scheme can save lots of running time, due to it provides a strategy to avert the solve the nonlinear algebraic equations. Thus, it is especially suitable for simulating a high-dimensions nonlinear oscillator containing fractional derivatives. With such capability in solving highly-dimensional systems, the presented method was further extended to solve a time fractional nonlinear diffusion-wave equation.

\section{ACKNOWLEDGMENTS}

This work is supported by National Natural Science Foundation of China (11572356, 11672337), and Guangdong Province Natural Science Foundation (1414050000412).

\section{REFERENCES}

[1] L. Bagley and J. Torvik, "A theoretical basis for the application of fractional calculus to viscoelasticity,” J. Rheol., 1983, 27(3), 201-210.

[2] C. Meral, J. Royston, and R. Magin, "Fractional calculus in viscoelasticity: an experimental study," Commun. Nonlinear Sci. Numer. Simulat., 2010 , 15(4), 939-945. 
[3] W. Chen, "An intuitive study of fractional derivative modeling and fractional quantum in soft matter.,"J. Vib. Control., 2008, 14(9-10), 16511657.

[4] S. Nicolle, P. Vezin, and F. Palierne, "A strain-hardening bi-power law for the nonlinear behaviour of biological soft tissues," J. Biomech., 2010, 43(5), 927-932.

[5] R. Metzler and J. Klafter, "The random walk's guide to anomalous diffusion: a fractional dynamics approach," Phys. Rep., 2000, 339(1), 177.

[6] A. Zhokh, I. Trypolskyi, and E. Strizhak, "An investigation of anomalous time-fractional diffusion of isopropyl alcohol in mesoporous silica," Int. J. Heat Mass Tran., 2017, 104, 493-502.

[7] I. Podlubny, "Fractional-order systems and PI-lambda-D-mu-controllers," IEEE T. Automat. Contr., 1999, 44(1), 208-214.

[8] A. Monje, Q. Chen, M. Vinagre, D. Xue, and V. Feliu-Batlle, "Fractionalorder systems and controls: fundamentals and applications," Springer Science \& Business Media, 2010

[9] J. Sabatier, P. Agrawal, and T. Machado, "Advances in Fractional Calculus," Springer, Dordrecht, 2007

[10] Q. Yang, D. Chen, T. Zhao, and Y. Chen, "Fractional calculus in image processing: a review," Fract. Calc. Appl. Anal, 2016, 19(5), 1222-1249.

[11] A. Rossikhin, and V. Shitikova, "Application of fractional calculus for dynamic problems of solid mechanics: novel trends and recent results," Appl. Mech. Rev. 2010, 63(1), 010801.

[12] D. Baleanu, H. Asad, and I. Petras, "Numerical solution of the fractional Euler-Lagrange's equations of a thin elastica model," Nonlinear Dyn, 2015, 81(1-2), 97-102.

[13] K. Diethelm, J. Ford, and D. Freed, "A predictor-corrector approach for the numerical solution of fractional differential equations," Nonlinear Dyn. 2001, 29(1), 3-22.

[14] K. Diethelm, and J. Ford, "Multi-order fractional differential equations and their numerical solution," Appl. Math. Comput, 2004, 154(3), 621640.

[15] P. Kumar, and P. Agrawal, "An approximate method for numerical solution of fractional differential equations," Signal Process, 2006, 86(10), 2602-2610.

[16] J. Huang, Y. Tang, and L. Vázquez, "Convergence analysis of a block-byblock method for fractional differential equations," Numer. Math-Theory. Me, 2012, 5(02), 229-241.

[17] J. Cao, and C.Xu, "A high order schema for the numerical solution of the fractional ordinary differential equations," J. Comput. Phys, 2013, 238, 154-168.

[18] C. Li, A. Chen, and J. Ye, "Numerical approaches to fractional calculus and fractional ordinary differential equation," J. Comput. Phys, 2011 230(9), 3352-3368.

[19] S. Yaghoobi, P. Moghaddam, and K. Ivaz, “An efficient cubic spline approximation for variable-order fractional differential equations with time delay," Nonlinear Dyn, 2017, 87(2), 815-826.

[20] T. Edwards, J. Ford, and C. Simpson, "The numerical solution of linear multi-term fractional differential equations: systems of equations," J. Comput. Appl. Math., 2002, 148(2), 401-418.

[21] Q. X. Liu, J. K. Liu, and Y. M. Chen, "An explicit hybrid method for multi-term fractional differential equations based on Adams and RungeKutta schemes," Nonlinear Dyn., 2016, 84(4), 2195-2203.

[22] Y. Xu, Y. Li, D. Liu, W. Jia, and H. Huang, "Responses of Duffing oscillator with fractional damping and random phase," Nonlinear Dyn., 2013, 74(3), 745-753.

[23] A. Schmidt, and L. Gaul, "Finite element formulation of viscoelastic constitutive equations using fractional time derivatives," Nonlinear Dyn., 2002, 29(1), 37-55.

[24] B. Yuste, and J. Quintana-Murillo, "Fast, accurate and robust adaptive finite difference methods for fractional diffusion equations," Numer. Algorithms., 2016, 71(1), 207-228.

[25] H. Doha, H. Bhrawy, and S. Ezz-Eldien, "Efficient Chebyshev spectral methods for solving multi-term fractional orders differential equations," Appl. Math. Model., 2011, 35(12), 5662-5672.
[26] W. Zhang, and N. Shimizu, "Numerical Algorithm for Dynamic Problems Involving Fractional Operators,” JSME Int. J. C., 1998, 41(3): 364-370.

[27] C. Galucio, F. Deü, S. Mengué, and F. Dubois, "An adaptation of the Gear scheme for fractional derivatives," Comput. Method. Appl. M., 2006 195(44), 6073-6085.

[28] C. Bucher, and A. Pirrotta, "Dynamic Finite Element analysis of fractionally damped structural systems in the time domain," Acta. Mech., 2015, 226(12), 3977

[29] F. Deü, and D. Matignon, "Simulation of fractionally damped mechanical systems by means of a Newmark-diffusive scheme," Comput. Math. Appl., 2010, 59(5), 1745-1753.

[30] D. Spanos, and I. Evangelatos, "Response of a non-linear system with restoring forces governed by fractional derivatives-Time domain simulation and statistical linearization solution," Soil. Dyn. Earthq. Eng., 2010, 30(9), 811-821.

[31] Q. X. Liu, J. K. Liu, and Y. M. Chen, "A second-order scheme for nonlinear fractional oscillators based on an Adams and Newmark- $\beta$ algorithms," Journal of Computational and Nonlinear Dynamics, DOI: http://computationalnonlinear.asmedigitalcollection.asme.org/acceptedm anuscripts.aspx. (accepted)

[32] A. Rossikhin, and V. Shitikova, "Application of Fractional Calculus for Dynamic Problems of Solid Mechanics: Novel Trends and Recent Results," Appl. Mech. Rev., 2010, 63(1), 010801.

[33] M. Dehghan, M. Abbaszadeh, and A. Mohebbi, "An implicit RBF meshless approach for solving the time fractional nonlinear sine-Gordon and Klein-Gordon equations," Eng. Anal. Bound. Elem., 2015, 50, 412 434 